\title{
La paz imposible en el proceso de evaluación de estudiantes con discapacidad intelectual leve en el Centro de Atención a Estudiantes con Discapacidad (CAED) del nivel media superior
}

Lic. en Psic. Cristian Díaz-Pedraza

pedraza_cristian@hotmail.com

México

https://orcid.org/0000-0002-2103-6024

\author{
Dr. en Ed. Carlos Israel Hannz-Sámano \\ hannzcarlos@gmail.com \\ México \\ https://orcid.org/0000-0001-5783-6835
}

Recepción:16 de febrero del 2021

Aprobación:28 de mayo del 2021

Publicación: 06 de junio del 2021

\section{Resumen}

El presente artículo explícita una descripción analítica respecto a la violencia estructural que se gesta y desarrolla en el proceso de evaluación por certificación dirigido a estudiantes con discapacidad intelectual leve del nivel educativo media superior adscritos al Centro de Atención a Estudiantes con Discapacidad (CAED) N. 065 ubicado en la ciudad de Toluca Estado de México.

El trabajo sustenta sus bases en un marco de investigación con enfoque de corte cualitativo, con nivel de estudio tipo exploratorio, mismo que se desarrolló bajo la perspectiva teórica de paz imposible del Doctor en sociología Eduardo Andrés Sandoval Forero, el cual señala desde una postura teórica el análisis de la violencia que dimana en el contexto de América Latina en sus dimensiones: estructural, directa, cultural, simbólica y ecológica. En tanto, este postulado se abordó desde una visión de paz integral que se acompaña bajo la directriz metodológica denominada Etnografía para la Paz (EtnoPaz).

La sistematización teórico-metodológica del método EtnoPaz en cada una de sus fases, permitió a partir del conocimiento empírico de campo, rescatar el pensar, sentir y actuar del sujeto de estudio mediante la aplicación de técnicas e instrumentos de investigación, 
situación que coadyuvó a conocer la realidad educativa del centro en interrelación con los diferentes actores educativos: auxiliar administrativo, asesores educativos, estudiantes con discapacidad intelectual leve y padres de familia.

Los resultados de la investigación se focalizan en conocer las condiciones de paz imposible que dimanan del proceso evaluativo dirigido al estudiante con discapacidad intelectual leve adscrito al CAED N. 065, y así, la gestión de espacios y centros educativos en igualdad y equidad social bajo los principios de paz integral.

Palabras clave: Paz, Educación, Evaluación, Discapacidad.

\title{
The impossible peace in the evaluation process of students with mild intellectual disabilities in the "Centro de Atención a Estudiantes con Discapacidad (CAED)" high-school
}

\begin{abstract}
This article presents an analytic description on the structural violence that develops and takes place on the evaluation process through certification aimed at high school level students who have a minor intellectual disability and who are part of the "Centro de Atención a Estudiantes con Discapacidad (CAED) No. 65)" located in the city of Toluca, Estado de México.
\end{abstract}

This work is backed up by a research frame with a qualitative focus and an exploratory study that was developed under the perspective theory of "impossible peace" from the PhD in Sociology Andrés Sandoval Forero, who points out — from a theoretical view - the analysis of the violence that surges from the Latin American context in different perspectives: structural, direct, cultural, symbolic and ecological. This approach was addressed from a vision of integral peace, shoulder to shoulder with the methodological guideline of Ethnography for Peace (EtnoPaz).

The theoretical-methodological systematization of the "EtnoPaz", in every of its stages, allowed — from the field empirical knowledge — to rescue the thoughts, feelings and performance of the subject of study through the application of instruments and investigation 
techniques. This situation allowed to get into touch with the educative reality inside the school and its interrelation with the different institutional actors: administrative assistants, tutors, students with minor intellectual disability and parents.

The results of this research are focused on the exploration of the conditions of "Impossible Peace" that surge from the evaluative process aimed at the student's evaluative process with minor intellectual disability from the CADEN No. 065 so that spaces and educative centers can be managed under the precepts of social equality and equity and the principles of integral peace.

\section{Keywords: Peace, Education, Evaluation, Disability.}

\section{Introducción}

El siglo XXI se encuentra estructurado por procesos de cambio político que impactan a nivel global dentro de las esferas: social, cultural, económica, educativa, entre otras, en el orbe mundial y en cada nación; dicha transformación socioeconómica ha legitimado a cada Estado-Nación para adoptar medidas in situ necesarias para mejorar la calidad de vida e índice de desarrollo humano entre sus habitantes.

En países de economía emergente como México, la transición sociopolítica en materia educativa se refleja en la promulgación de ordenamientos jurídico-normativos que garanticen a sus ciudadanos una educación de calidad que van más allá de la formación académica centrada en los procesos de enseñanza-aprendizaje, sino más bien en normas jurídicas heterónomas, es decir bajo preceptos del Estado, donde el sistema educativo se rige por coacción y se establece como imperante.

En este sentido, los estudios para la paz en el ámbito educativo forman parte de uno de los tópicos que coadyuvan a comprender las diversas formas de expresión, actuación e interrelación al interior escolar para mantener relaciones dialógicas, ecuánimes y libres de violencia que permitan construir una nueva cosmovisión social educativa centrada en el stricto sensu al respeto de la diversidad. 
Es bajo esta línea temática que, en México, se dilucida en materia educativa prevalecen estrategias gubernamentales de carácter político para el fomento de una educación para la paz, dentro del cual emergen diversas organizaciones escolares en el país, como los Centros de Atención a Estudiantes con Discapacidad (CAED), cuya esencia radica en fortalecer en acompañamiento el proceso académico de estudiantes con discapacidad.

La coexistencia de reformas estructurales en política educativa del país, ha determinado establecer diversos CAED a partir de 2009, estos centros educativos se fundamentan legalmente desde la Constitución Política de los Estado Unidos Mexicanos (CPEUM) y la Ley General de Educación; en lo académico, con base en un currículo, el cual contempla áreas sociales, psicopedagógicas, filosóficas, epistemológicas, e institucionales, donde la estructura teórica y metodológica se ciñe dentro la formación y preparación a estudiantes con discapacidad en el nivel medio superior por medio de evaluaciones por certificación y lograr el egreso.

En descripción del contexto educativo de los centros, la situación es irregular específicamente en el proceso de evaluación por certificación a estudiantes con discapacidad, en otras palabras, se observa violencia estructural en la aplicación de exámenes, por desconocimiento de la discapacidad en la trasposición didáctica.

En perspectiva teórica de Sandoval (2018a), se dilucida que en el caso CAED N. 065, coexiste paz imposible inmersa en el proceso de evaluación que afecta directamente a los diferentes actores educativos con discapacidad intelectual leve adscritos a dicho centro.

La reflexión ante la problemática del CAED N. 065 en este referente, requiere de una participación social y educativa más consciente e informada que permita responder a las necesidades de una población con discapacidad intelectual leve, cuyos derechos humanos y constitucionales son vulnerados en tanto prevalezca la violencia estructural.

En este sentido, el objetivo de la presente investigación en perspectiva teórica de paz imposible es un elemento clave para el análisis reflexivo donde se denota la persistencia de violencia estructural como parte del proceso de evaluación dirigido a estudiantes con discapacidad del nivel educativo media superior adscritos al CAED N. 065.

El escrito se desglosa en dos apartados bajo seis ejes temáticos: 


\section{Estructura Teórica-Normativa}

1.1. Centros de Atención a Estudiantes con Discapacidad (CAED).

1.2. Proceso de evaluación en educación media superior.

1.3. Generalidades de la Paz Integral.

2. Marco metodológico

2.1. Metodología.

2.2. Proceso metodológico.

2.3. Resultados.

Conclusiones.

Fuentes de consulta.

La investigación se postuló como parte la Maestría en Interculturalidad para la Paz y los Conflictos Escolares (MIPCE), que se desarrolló en la Centenaria y Benemérita Escuela Normal para Profesores de Toluca (CyBENP).

\section{Estructura Teórica-Normativa}

\subsection{Centros de Atención a Estudiantes con Discapacidad (CAED).}

En el ámbito educativo, ha sido imprescindible la eliminación de barreras para el aprendizaje y participación de las personas con discapacidad, con la finalidad de construir una sociedad bajo los preceptos de equidad e imparcialidad, alejada de prácticas discriminatorias, ante este contexto, prevalecen programas a nivel nacional, que enmarcan, acuerdos políticogubernamentales que salvaguardan la integridad y derechos humanos de toda persona.

En México, el año fiscal 2008, marcó un hito que suscitó importantes cambios en propuestas educativas del país, el gobierno federal destinó recursos económicos a nivel nacional para la creación de instituciones en el nivel media superior de carácter público, bajo el propósito fundamental de priorizar una educación inclusiva hacia los sectores de la población en riesgo de vulnerabilidad.

El Gobierno de la República Mexicana, como parte de las iniciativas de la Secretaría de Educación Pública (SEP) a través de los decretos: Plan Nacional de Desarrollo (PND) 2013-2018, 2019-2024; Programa Sectorial de Educación (PSE) 2013-2018; y, Programa para la Inclusión y Equidad Educativa (PIEE), 2013-2018, 2019-2024, establecen las 
estrategias pertinentes para brindar atención educativa oportuna, a jóvenes con discapacidad para cursar el bachillerato.

Los documentos con fecha de emisión 2019-2024, dan continuidad a las acciones de los Centros de Atención para Estudiantes con Discapacidad (CAED), en tanto los salvoconductos precedentes 2013-2018 plasman los criterios para la creación de estos, conocidos a su vez, según el Acuerdo número 24/12/14 como: "Aulas Gilberto Rincón Gallardo", en enaltecimiento a dicho personaje, quien en pugna por las garantías individuales de las personas con discapacidad fungió como dirigente del Consejo Nacional para Prevenir la Discriminación (CONAPRED) (Diario Oficial de la Federación de México a 17 de diciembre de 2014).

Es en este referente que los CAED según el ACUERDO número 24/12/14 inician su operación en el año 2009 con "46 [...] [instituciones] en las principales ciudades del país”, de las cuales a septiembre de 2020 con base en datos estadísticos de la página ENLACE CAED, existen 291 planteles, ubicados en los 32 estados de la república mexicana (Diario Oficial de la Federación de México 27 de diciembre 2014).

\subsection{Proceso de evaluación en educación media superior}

La evaluación del aprendizaje como instrumento de apoyo dentro del contexto educativo media superior juega un papel preponderante que coadyuva a través de la literatura a reconocer su acepción teórica como una "[...] etapa del proceso de enseñanza-aprendizaje que se utiliza para detectar el progreso del alumno” (López e Hinojosa, 2005, p. 13).

En otra mirada Castillo y Cabrerizo (2010) refieren que la evaluación se establece a partir de "[...] un conjunto de acciones que deben sucederse a lo largo del desarrollo de los procesos de aprendizaje y de enseñanza para que los alumnos puedan conseguir los objetivos que se pretenden y las competencias" (p.129).

La paralaje teórica de ambas posturas permite entrever que la evaluación se encuentra vinculada a las actividades que se generan durante el proceso de enseñanza-aprendizaje, en el que coexisten dos agentes educativos: evaluado; y evaluador; al cual corresponde la certificación de competencias del evaluado a partir de la aplicación de un instrumento para cualificar el desempeño escolar. 
En este escenario Tobón, Pimienta y García (2010) argumentan que el proceso de evaluación de las competencias "Se determina considerando los tipos, finalidades y participantes en la evaluación" (véase tabla 1) bajo lo siguiente:

\section{Tabla 1.}

Proceso de evaluación por competencias

\begin{tabular}{|c|c|}
\hline Tipos de evaluación & $\begin{array}{l}\text { Diagnóstica } \\
\text { Formativa }\end{array}$ \\
\hline Finalidades de la evaluación & $\begin{array}{l}\text { Promoción } \\
\text { Acreditación } \\
\text { Certificación }\end{array}$ \\
\hline Participantes en la evaluación & $\begin{array}{l}\text { Autoevaluación } \\
\text { Coevaluación } \\
\text { Heteroevaluación }\end{array}$ \\
\hline
\end{tabular}

En este entender y para fines del presente escrito, se hace referencia al modelo de Tobón, Pimienta y García (2010) sobre el proceso de evaluación por competencias de los estudiantes con discapacidad adscritos al CAED N. 065, el cual se analiza desde una vertiente teórica en dos apartados que enfatizan en el tipo de evaluación que se genera tanto de forma externa como interna para evaluar el aprendizaje:

1.2.1. El primero alude al tipo de evaluación que se genera de forma externa para la acreditación modular de los estudiantes con discapacidad desde los planteamientos normativos.

1.2.2. El segundo aborda el tipo de evaluación que se genera al interior áulico para desarrolló de competencias en el estudiante con discapacidad.

\subsubsection{Evaluación externa}

Los Centros de Atención a Estudiantes con Discapacidad (CAED), según el Acuerdo número 18/09/19 son unidades centralizadas de la Subsecretaria de Educación Media Superior (SEMS), dependientes de la Dirección General de Bachillerato (DGB) que se guían bajo los 
lineamientos y acuerdos normativos establecidos en la Reforma Integral de Educación Media Superior (RIEMS) (Diario Oficial de la Federación de México a 20 de septiembre de 2019).

Este primer apartado, con base en datos de la DGB (2017, pp. 11-16) permitió situar al CAED como un servicio educativo de bachillerato general no escolarizado dependiente de la Dirección de Sistemas Abiertos (DSA), que opera bajó: enfoque constructivista; modelo en competencias; y un plan modular de 22 asignaturas que el estudiante deberá acreditar mediante certificación por evaluaciones parciales para concluir sus estudios.

El CAED al ser parte de un sistema no escolarizado se encuentra alineado al Acuerdo Secretarial de la RIEMS número 445, por el que se conceptualizan y definen para la Educación Media Superior las opciones educativas en las diferentes modalidades, el cual establece, en su ARTICULO CUARTO; inciso VI; que el tipo de evaluación acorde a esta modalidad educativa corresponde a la "Certificación por Evaluaciones Parciales" (Diario Oficial de la Federación de México a 21 de octubre de 2008).

La normatividad en materia de evaluación que refiere la DGB (2017) para el programa CAED, se circunscribe a la acreditación de 22 módulos establecidos en su plan de estudios, a través de la aplicación de exámenes de carácter externo por asignatura, mediante la opción de Certificación por Evaluaciones Parciales que establece como parte del Acuerdo RIEMS número 445 para el nivel media superior.

En este sentido se dilucida que la evaluación de los estudiantes con discapacidad adscritos al CAED N. 065 alude a un proceso de certificación que se circunscribe a la valoración de los aprendizajes por competencias a través de un instrumento tipo examen que se aplica de forma externa para conocer los avances académicos respecto a uno de 22 módulos establecidos en el plan de estudios.

En relación al escenario que presenta el centro en materia de certificación y con base al proceso de evaluación por competencias de Tobón, Pimienta y García (2010), se elucida considerando la finalidad evaluativa, que los estudiantes con discapacidad CAED N. 065 se evalúan de forma externa con la finalidad de acreditación y certificación, ante lo cual citan textualmente: 
- [La] Evaluación de acreditación: se hace al final de un módulo para reconocer las competencias de los estudiantes en un nivel determinado.

- [En tanto, la] Evaluación de certificación: se hace para certificar las competencias por parte de organizaciones competentes para ello (p. 133).

El proceso de evaluación para acreditación en este sentido se realiza periódicamente por los estudiantes con discapacidad adscritos al CAED N. 065, con la finalidad de acreditar uno de 22 módulos.

En tanto la evaluación por certificación permite a la DGB en colaborativo con el Departamento de Procesos de Información (DPI) de la DSA como autoridades educativas competentes encargadas de la aplicación externa del examen, verificar que las competencias estudiantiles se alcanzaron.

En lo que respecta a la evaluación externa en paráfrasis con Ravela (2006), se argumenta es una evaluación de carácter estandarizada que "[... ] sirve para poner el foco de atención en aquello que todos los alumnos deberían aprender, pero, por supuesto, no puede ni pretende dar cuenta de todos los aprendizajes" (p. 73).

En este sentido podemos afirmar que una de las particularidades de la evaluación externa para el nivel educativo media superior CAED N. 065 radica en su carácter homogéneo, a través del cual se evalúa a una población de estudiantes con discapacidad: auditiva, visual, motriz e intelectual bajo estándares generales.

En apoyo a este argumento, el Instituto Nacional para la Evaluación (INNE, 2019) refiere que las evaluaciones a gran escala se encuentran constituidas por "[...] altos niveles de estandarización, tanto en el diseño de los instrumentos como en su aplicación, con el fin de garantizar la comparabilidad de los resultados" (p.7).

\subsubsection{La evaluación interna}

Este segundo apartado hace alusión al proceso de evaluación que se generó de manera interna para evaluar el aprendizaje de los estudiantes con discapacidad, el cual deriva de las asesorías que el personal adscrito al CAED N. 065 lleva a cabo, para ello se explica en sucinto el principio por el cual emerge el programa, así como las funciones: de asesoría; y del asesor.

El CAED como programa único a nivel nacional de tipo media superior, sustenta su principio rector en los Mecanismos Operativos CAED (DGB, 2017) con base en la Estrategia 
3.5 del PND 2013-2018, de "Impulsar nuevas formas y espacios de atención educativa para la inclusión de las personas con discapacidad [...]" (Diario Oficial de la Federación de México a 13 de diciembre de 2013).

Es bajo este planteamiento que se despliegan las líneas normativas a través de un modelo educativo no escolarizado de "Mediación docente [que brinda asesoría educativa] En función de las necesidades del estudiante" con discapacidad. (DGB, 2017, p. 11). Según el ACUERDO número 18/09/2019 por el que se modifican las Reglas de Operación del Programa para la Inclusión y la Equidad Educativa para el ejercicio fiscal 2019, emitidas mediante diverso número 04/02/19 publicado el 28 de febrero de 2019, la acepción teórica de asesoría educativa hace referencia al:

Proceso de facilitación y acompañamiento del aprendizaje de las/os alumnas o alumnos con discapacidad, desde que se incorporan hasta que completan sus estudios de tipo medio superior, mediante el cual se procura que las personas reflexionen, resuelvan dudas y reciban retroalimentación. Puede darse en los Centros de Atención para Estudiantes con Discapacidad (CAED) (Diario Oficial de la Federación de México a 20 de septiembre de 2019).

El postulado refleja el trabajo concerniente a los asesores CAED N. 065 mediante el cual se prioriza una atención educativa que permite guiar a los estudiantes con discapacidad durante su trayecto académico a través de una interacción dialógica y reflexiva para el aprendizaje. Estos elementos clave como parte de las funciones específicas a desarrollar por el asesor durante el proceso de enseñanza, le permiten a este:

Funciones específicas

- Elaborar y apoyar a los estudiantes en el manejo de material didáctico orientado adaptado a las necesidades de los estudiantes y apegado a los contenidos temáticos y los libros de texto de Preparatoria Abierta.

- Brindar orientación académica de acuerdo al Plan de Estudios de Preparatoria Abierta y en función de las necesidades específicas que representa cada tipo de discapacidad.

$[\ldots]$

- Dar a conocer a los estudiantes el programa de la asignatura, materiales didácticos y las formas de evaluación al inicio del servicio académico.

$[\ldots]$

- Realizar evaluaciones para apoyar el aprendizaje de los estudiantes de los CAED (DGB, 2017, p.26). 
Este referente se circunscribe en las funciones específicas del asesor educativo CAED N. 065, ante el cual queda a cargo en materia de evaluación: a) diagnóstica; notificar al estudiante sobre la valoración inicial por asignatura, y b) formativa; orientar y apoyar el proceso de aprendizaje a través del diseño diversificado de material didáctico por tipo de discapacidad en apego a los lineamientos normativos del programa.

En este sentido, la evaluación inicial o diagnóstica, juega un papel preponderante en el contexto educativo CAED N. 065, debido a que permite obtener al asesor educativo un panorama amplio sobre los conocimientos previos del estudiante al momento de emprender nuevos proyectos académicos sobre cada uno de los 22 módulos correspondientes al plan de estudios.

En este referente, algunos autores como Tobón, Pimienta y García (2010) afirman que la evaluación diagnóstica se realiza al "inicio de un proceso" (p. 133), educativo con la finalidad de valorar los conocimientos previos del estudiante, debido a que esta "[...] permite saber, entre otras cosas, cuál es el estado cognoscitivo y actitudinal de los alumnos [...] [para] ajustar la acción a las características de los alumnos, a su peculiar situación" (Santos, 1995, p. 63).

La paralaje teórica de los autores respecto al tipo de evaluación: diagnóstica contribuye a dilucidar que el proceso de valoración inicial en el CAED N. 065 permitió al asesor educativo realizar una valoración inicial del conocimiento estudiantil con la finalidad de recabar datos sustanciales durante el trayecto de enseñanza aprendizaje, para una posterior intervención.

En tanto, el proceso de evaluación concerniente al tipo formativo según Casanova (1998):

[...] se utiliza en la valoración de procesos ([...] de enseñanza aprendizaje...) y supone, por lo tanto, la obtención rigurosa de datos a lo largo de ese mismo proceso, de modo que en todo momento se posea el conocimiento apropiado de la situación evaluada [...] Su finalidad, [...] es mejorar o perfeccionar el proceso que se evalúa (p. 81).

Revista RedCA, Vol. 4, Núm. 10 (junio-septiembre 2021) Universidad Autónoma del Estado de México, ISSN-e: 2594-2824 
En otra mirada López e Hinojosa (2005) plantean que "La evaluación formativa se obtiene durante el proceso de enseñanza-aprendizaje, para mejorarlo y dirigirlo por una serie de pasos de retroalimentación constante" (p. 27), que coadyuven al estudiante a alcanzar los propósitos de aprendizaje. En esta misma perspectiva Tobón, Pimienta y García (2010) refieren que la evaluación formativa "se hace durante el proceso. [La cual] No implica acreditación académica de las competencias" (p. 148).

La perspectiva teórica de los autores respecto a evaluación formativa alude al proceso dentro del ámbito educativo CAED N. 065 que se realiza durante el trayecto académico sobre cada uno de los 22 módulos del plan de estudios, a través del cual se conduce y retroalimenta la enseñanza-aprendizaje mediante la diversificación de material didáctico, con la finalidad coadyuvar al estudiante a alcanzar los propósitos educativos.

\subsection{Generalidades de la Paz Integral}

\subsubsection{Estudios para la Paz}

El siglo XX como antecedente sociohistórico de estudios para la paz en paráfrasis con Sandoval (2016b), conllevó a situar su ontogénesis conceptual posterior a las confrontaciones bélicas de la Segunda Guerra Mundial, situación que a su término permitió “[ [...] construir nuevas formas más pacíficas y menos violentas de relacionarse” (p. 110).

Este pensar preventivo de reincidencia ante nuevos sucesos bélicos, coadyuvó en su pugna por erradicar actos que subyugaran la integridad social, a proclamar en 1948 la Declaración Universal de los Derechos Humanos como salvoconducto de orden normativo mundial.

La emisión del documento “[...] hizo evidente que la protección internacional de los derechos humanos era una condición esencial para la paz y el progreso de la humanidad" (Del Toro, 2012, p. 32) en tanto, introdujo las garantías individuales para salvaguardar la integridad, libertad y justicia en las diferentes esferas sociales: política; económica; cultural; y educativa.

Este acontecimiento sociohistórico inspiró a diferentes pensadores en el contexto de Occidente y Norteamérica, los cuales se sumaron en prospectiva, al desarrollo de propuestas 
teóricas de paz, bajo una nueva cosmovisión para comprender e interpretar sucesos violentos, así como la búsqueda pacífica de acciones para la resolución de conflictos.

En este sentido Jares (1999) y Sandoval (2016b) bajo la misma paralaje ideológica, afirman que algunos teóricos que contribuyeron a través de sus planteamientos a generar una nueva cosmovisión de paz posterior a la Segunda Guerra Mundial son el sociólogo Johan Galtung; el filósofo Vicent Martínez Guzmán; y el historiador Francisco A. Muñoz.

Es bajo este referente que la Investigación para la Paz (IP), según Cobalti (1985) citado por Jares (1999, p.81) “[...] se puede considerar, así, un producto de la Segunda Guerra Mundial”, la cual, según el autor, permitió reformular la acepción de estudios para la paz, de conflicto, así como la visión de educación para la paz.

La investigación desde la perspectiva de estos pensadores, a pesar de ser un referente teórico mundial en estudios para la paz, se sitúa según Sandoval (2016b) bajo “[...] perspectivas liberales eurocéntricas-norteamericanas teóricas de la paz positiva, la paz negativa, hacer las paces, la paz imperfecta o la paz neutra" (p.43).

En paráfrasis autoral, se plantea dichos estudios emergen como base teórica en un tejido social específico que dista de los problemas presentes en Latinoamérica, es por ello, que sitúa la imperante de análisis reflexiva desde el postulado teórico de paz integral, con la finalidad de responder a los conflictos y violencias propios de cada contexto.

\subsubsection{Paz integral}

La paz integral como constructo teórico, se gesta desde los estudios para la paz a partir de la perspectiva teórica del Sociólogo Andrés Sandoval Forero con la finalidad de analizar la violencia y conflictos inmersos en el contexto de América Latina. Su acepción se asume como una paz:

[...] activa, noviolenta y duradera, es decir una paz sustentable, a partir de un re-enfoque que tenga como base, por un lado, el conocimiento de nuestras realidades, y por otro, referentes hipotéticos de las teorías del pensamiento crítico latinoamericano en cualquiera de sus expresiones (Sandoval, 2016b, p.44). 
Este planteamiento deja entrever que el análisis de paz en el contexto latinoamericano debe situarse desde la necesidad local propia de cada estudio, el cual debe sustentarse en referentes teóricos cuya crítica permita la reflexión para "descolonizarnos en el pensar y en la praxis de paz" (Sandoval, 2016b, p.49). Es por ello, que la paz integral se presenta como:

[...] el antídoto de condiciones insostenibles para la paz, y los fundamentos teóricos que describen la paz imposible se encuentran en la persistencia de las violencias físicas, y culturales que se anidan con la violencia estructural conformada por la pobreza, la marginación y la exclusión social y educativa, es decir condiciones determinadas por las violencias sistémicas (Sandoval, 2016b, p. 50).

La paz integral en este sentido se muestra desde un panorama global que tiene como propósito fundamental erradicar los diferentes tipos de violencia inmersos en la esfera social bajo los principios de "[...] justicia, libertad, respeto a los derechos humanos, a los derechos de los pueblos indígenas, a la autonomía, interculturalidad y democracias reales" (Sandoval, 2016b, p.49).

Es bajo este referente teórico y con fines de la presente investigación que se retoma el enfoque de paz integral, con la finalidad de hacer un análisis reflexivo desde los planteamientos para la paz, sobre la situación de violencia que emerge del contexto educativo CAED N. 065.

\subsubsection{Paz imposible}

La gestión de espacios educativos desde un enfoque teórico de paz integral según Sandoval (2016b) sitúa un análisis contextual en el cual converge la violencia en sus dimensiones: a) estructural, b) directa, c) cultural, d) simbólica y e) ecológica, dentro de una vertiente macroestructural que fractura en subsecuente, las relaciones pacíficas existentes en los microsistemas, en este sentido el autor afirma que la:

[...] Paz Integral tiene como supuesto que la paz es imposible mientras tenga presencia la violencia en alguna de las modalidades o tipologías [...] [por lo que se vuelve necesario desde] La propuesta de la integralidad para la paz [...] construir en la dimensión estructural, cultural y simbólica, así como en el ámbito social, educativo y familiar, condiciones de convivencia dignas, justas y pacíficas que garanticen que la paz sea sustentable y duradera (p. 143).

El postulado teórico permite dilucidar que la paz imposible se constituye por una serie

de violencias que convergen entre sí, las cuales se presentan dentro de un contexto 
determinado a través de diversas formas que ciñen la coexistencia de espacios armónicos y de igualdad social para el desarrollo pleno del ser humano (véase figura 1).

Figura 1.

Paz imposible

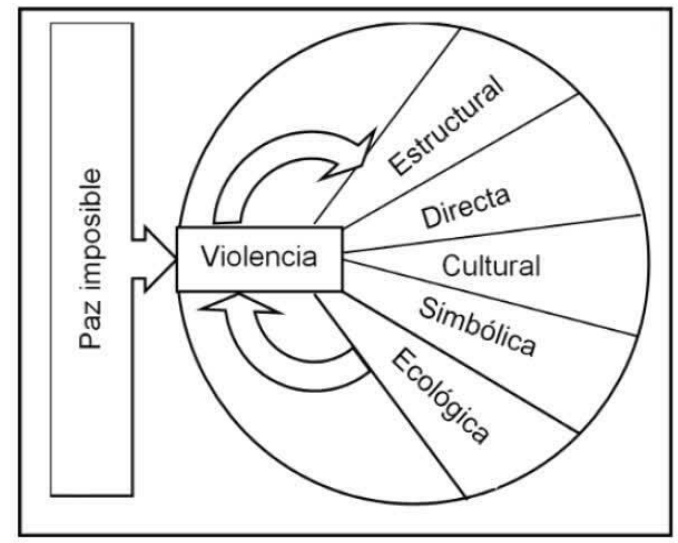

Fuente: Elaboración propia con base Sandoval (2016b, pp.92-97) y Salazar (2018a, p. 51)

En apoyo a este argumento Salazar (2018b) afirma que la paz imposible debe explicarse "por medio de los indicadores de violencia sistémica, violencia estructural, violencia simbólica, violencia cultural [...] violencia física" y ecológica presentes en el contexto (p.50).

Es a partir de estos planteamientos y para fines del presente escrito que se genera un análisis de la paz imposible desde la vertiente de violencia estructural inmersa en el proceso de evaluación por certificación de los estudiantes con discapacidad intelectual leve, en sistema educativo CAED N. 065.

\subsubsection{Violencia estructural}


La acepción de violencia estructural desde la perspectiva teórica-conceptual de paz imposible se define según Sandoval (2016b) como "aquella [violencia] que viene del Estado, de las leyes y de las instituciones en general. Son estructuras económicas, jurídicas y políticas del Estado generadoras de opresión que impiden la libertad del ser humano” (pp. 111-112).

En respecto a este planteamiento, la violencia estructural se circunscribe como un proceso que ciñe el desarrollo del ser humano en sus diferentes ámbitos y el cual emerge como medio de dominación por parte de las organizaciones centralizadas y descentralizadas provenientes de la esfera político-gubernamental manifiesta a través de políticas públicas y de Estado.

En otra mirada, el acercamiento teórico al constructo de violencia estructural desde la perspectiva escolar, según Salazar (2014) citada por Salazar (2018b), se encuentra inscrito:

[...] en el marco social y su principal característica es la desigualdad. Se trata de una injusticia social que influye en niños y adolescentes, y se hace presente de manera directa o indirecta en las escuelas. Se refiere al conjunto de estructuras físicas y organizativas que no permiten la satisfacción humana (p.55).

Esta paralaje teórica, permite comprender que una de las características de la violencia en su dimensión estructural dentro del contexto escolar, se atribuye a las inequidades existentes y la cual puede presentarse a través múltiples formas que afectan a la población estudiantil en los distintos niveles del sistema educativo para satisfacer sus necesidades, así como el desarrollo académico de los mismos.

Es bajo estos postulados teóricos que la presente investigación en análisis de la violencia en su dimensión estructural inmersa en el proceso de evaluación por certificación dirigida a estudiantes con discapacidad intelectual leve adscritos al CAED N.065, retoma la perspectiva de paz imposible para su reflexión.

\section{Marco metodológico}

\subsection{Metodología}

El proyecto parte de un enfoque metodológico de corte cualitativo, el cual permitió comprender la realidad de los actores socioeducativos al interior del contexto escolar CAED 
N. 065. El nivel de investigación que se empleó fue el estudio de tipo exploratorio, en pretensión de generar nuevas líneas de investigación por ser un acercamiento fenómeno y ampliar la literatura existente al centro respecto a los tópicos: proceso de evaluación por certificación dirigido a estudiantes con discapacidad; y violencia estructural.

La investigación se llevó a cabo bajo la directriz del método: Etnografía para la Paz (EtnoPaz), como parte del "[...] nuevo paradigma de los estudios para la paz, que bien se puede particularizar [...] en los estudios de los conflictos, las violencias, la interculturalidad para la paz, la democracia, la diversidad étnica, social y cultural”' (Sandoval, 2018a, pp. 5253).

Este planteamiento en el campo investigativo conlleva desde una vertiente crítica a la gestión socioeducativa de ambientes de paz, en los que resulta imperante la decolonización del pensamiento para la deconstrucción, coproducción y reconstrucción de saberes que permitan interiorizar nuevas formas de interrelación social en igualdad de derechos.

La aplicación del método, el cual se estructura en siete fases (véase figura 2), permitió a partir del conocimiento empírico de campo, rescatar el pensar, sentir y actuar del sujeto de estudio a través del diálogo, el cuál mantiene una relación estrecha que se entrelaza con la parte teórica. La esquematización se muestra bajo la siguiente figura:

\section{Figura 2}

\section{Fases: Etnografía para la Paz}

1) $\begin{aligned} & \text { Precisión de lo que se va a investigar en cuanto a lo temático, lo } \\ & \text { espacial y lo temporal. }\end{aligned}$
actores sociales a partir de referentes empíricos.
3) $\begin{aligned} & \text { Selección adecuada de las técnicas, los instrumentos y las } \\ & \text { herramientas de investigación. }\end{aligned}$
4) $\begin{aligned} & \text { Clasificación, codificación y categorización de los datos de } \\ & \text { campo. }\end{aligned}$

Fuente: Elaboración propia con base en el texto de Sandoval (2013c, p. 22) Etnografía para la paz, la interculturalidad y los conflictos. 


\subsection{Proceso metodológico}

El proceso metodológico muestra la sistematización de información que deriva de la investigación, la cual considera las siete fases de Etnografía para la Paz, mismas que se describen en orden jerárquico bajo lo siguiente:

1) Precisión de lo que se va a investigar en cuanto a lo temático, lo espacial y lo temporal.

El estudio contemplo la investigación respecto al tópico "La paz imposible en el proceso de evaluación de estudiantes con discapacidad intelectual en el Centro de Atención a Estudiantes con Discapacidad (CAED) N. 065 del nivel medio superior. El proyecto tuvo lugar en la delegación de Santa Cruz Atzcapotzaltongo, Toluca Estado de México, de agosto 2018 a junio 2020.

2) Realización de etnografía de campo en coproducción con los actores sociales a partir de referentes empíricos.

La inmersión en el contexto educativo CAED N. 065, conllevó a establecer comunicación directa con la autoridad responsable del centro, a quien se solicitó el ingreso vía oficio, posterior a la aceptación, se procedió al análisis, registro e interpretación de datos en coproducción con actores socioeducativos.

3) Selección adecuada de las técnicas, los instrumentos y las herramientas de investigación.

La selección de técnicas e instrumentos de investigación se realizó con base en la metodología cualitativa, la cual se encuentra en correlación con el método EtnoPaz, esto permitió la recopilación e interpretación de información, desde la percepción de los actores socioeducativos del CAED N. 065 bajo lo siguiente:

a) Encuesta: instrumento tipo cuestionario mixto; se aplicó a 23 estudiantes con discapacidad intelectual leve inscritos al CAED N. 065, a partir del cual se recuperó información sobre los factores que afectan el proceso de evaluación.

b) Encuesta: instrumento tipo cuestionario con preguntas abiertas; se aplicó al personal CAED N. 065, que equivale a 4 de 3 personas, se rescata la opinión respecto al proceso de evaluación de 1; auxiliar administrativo y 2; asesores educativos. 
c) Entrevista telefónica semiestructurada: instrumento guion de entrevista; se aplicó a seis padres de familia electos de forma aleatoria, la conversación se grabó bajo autorización de estos, se recuperó la opinión respecto al proceso de evaluación del CAED N. 065.

d) Encuesta: instrumento denominado Evaluación Factorial de las Habilidades Intelectuales (EFAI-4); se aplicó a 20 estudiantes con discapacidad intelectual leve, se rescata información respecto a la aptitud, espacial, numérica, verbal, razonamiento abstracto y memoria del estudiante.

4) Clasificación, codificación y categorización de los datos de campo.

La recolección de datos a partir de las técnicas e instrumentos aplicados, coadyuvaron al análisis e interpretación de resultados a partir del planteamiento teórico de paz integral, los cuales se clasificaron con base en la unidad de observación: proceso de evaluación. Posterior a ello se codificó y categorizo mediante la teoría fundamentada o gran teoría con base en Murillo (s/f, pp. 24-27), el procedimiento se realizó en tres pasos:

a) Limpieza de datos; se verificó línea por línea la información plasmada en las técnicas e instrumentos, para su depuración.

b) Codificación abierta; permitió seleccionar y agrupar información iterativa para la construcción de códigos: abiertos e in vivo, de los cuales emergieron diferentes subcategorías.

c) Codificación axial; coadyuvó a identificar las subcategorías de análisis, las cuales se agruparon con base en el constructo teórico de paz imposible, del cual emerge la categoría central: violencia estructural.

5) Construcción conceptual y teórica de la información de campo.

La construcción conceptual y teórica respecto a la categoría central de análisis: violencia estructural; partió de un enfoque, analítico e investigativo, que se relaciona al trabajo a partir de los datos empíricos realizados en campo y se integra a la investigación desde el postulado de paz integral. 
En este sentido, se identificó que la violencia estructural dirigida a estudiantes con discapacidad intelectual leve adscritos al CAED N. 065 es uno de los factores que afectan de manera directa a la población bajo esta condición durante el proceso de evaluación por certificación, encontrándose presente en la omisión de estatutos legales y jurídico normativos para la construcción de instrumentos tipo examen acordes a las características cognitivas del estudiante, que le permitan ejercer en igualdad de oportunidad, sus derechos constitucionales y garantías individuales en materia educativa.

6) Cimentación de conocimiento teórico y analítico de realidades sobre la paz, la interculturalidad, los conflictos y la democracia.

El análisis de información sobre los datos del CAED N. 065 permitió organizar, categorizar, conceptualizar y explicar desde los fundamentos teóricos de paz integral, la realidad educativa del centro durante el proceso de evaluación por certificación, en el cual prevalece una interrelación de los hallazgos de campo, con la parte teórica.

El conocimiento teórico de la realidad CAED N. 065 sobre aspectos relacionados a la paz integral durante el proceso de evaluación por certificación es limitado y disperso, ya que, al presentarse paz imposible en su dimensión de violencia estructural, se ciñe la conjunción académica respecto al aprendizaje, lo que deriva en factores relacionados a exclusión educativa, rezago y abandono escolar que afecta en subsecuente a una población con discapacidad intelectual leve.

7) Elaboración del texto etnográfico.

El resultado sobre los hallazgos de campo se describe con base en el postulado de paz integral. El texto etnográfico muestra la perspectiva de los actores socioeducativos CAED N. 065: auxiliar administrativo, asesores educativos, estudiantes con discapacidad y padres de familia.

Los datos, conllevan a establecer la existencia de paz imposible dentro del contexto educativo, la cual se presenta durante el proceso de evaluación por certificaciones, a través de una violencia estructural que proviene del Estado y limita la en lo subsecuente la igualdad de derechos ante personas con discapacidad. 


\subsection{Resultados}

Los resultados de la investigación se integran por técnica e instrumento de aplicación, a partir de la percepción de los actores socioeducativos CAED N. 065 sobre el proceso de evaluación por certificación:

R1) Técnica encuesta: Percepción estudiantil sobre el proceso de evaluación CAED N. 065

El instrumento tipo cuestionario se diseñó y adapto con base en el Informe estadístico sobre las evaluaciones en CAED de Cortes, Gonzáles, Guerrero y Rivera (2016), el cual se dividió en tres apartados: I) Libros de texto; II) Estructura del examen; III) Respuestas del examen.

Los resultados de aplicación corresponden a 23 estudiantes con discapacidad intelectual leve inscritos al CAED N. 065, de los cuales se recupera información respecto a los factores que intervienen en la acreditación por examen, los apartados muestran:

I) Libros de texto. Recuperó la percepción el estudiante con discapacidad sobre la relación existente entre los contenidos del libro de texto correspondiente a uno de los 22 módulos del plan modular de estudios y las interrogantes que se establecen en la evaluación parcial que se presenta con fines de acreditación.

En respecto a este apartado, los estudiantes refieren que una de las dificultades a las que se han enfrentado al momento de dar respuesta a la evaluación por certificación, se relaciona a la divergencia temática que existe en los libros de texto que revisan durante asesoría, con los cuestionamientos que se establecen en el instrumento tipo examen, ante lo que manifiestan, se les ha interrogado sobre otros tópicos que difieren del módulo que se encuentran estudiando.

II) Estructura del examen. Permitió conocer la opinión del estudiante con discapacidad sobre el formato que presenta este, así como de los reactivos tipo opción múltiple de relación continuativa, de complementación, de negación, acompañados de gráficas, esquemas y lecturas extensas, que se le presentan al momento de ser evaluados.

Los resultados muestran que el estudiante con discapacidad intelectual leve tiene dificultad durante la lectura tipo del examen cuando se le presentan reactivos con 
palabras cuyo significado desconocen. Respecto a las preguntas de opción múltiple acompañados de gráficas o esquemas refieren se confunden al momento de interpretar o relacionar los datos.

En referente a las preguntas acompañadas de lecturas extensas mencionan existe dificultad para llevar a cabo una relación continuativa cuando se le presentan números o letras dentro de las respuestas. En tanto, con respecto a las preguntas de negación, afirman prevalece confusión al momento de seleccionar la respuesta correcta.

En lo que concierne a la estructura del examen, los estudiantes externan que tanto las preguntas como las respuestas de opción múltiple deben estar constituidas de forma sencilla, explicita y no tan compleja con la finalidad de que les permita comprender la información.

III) Respuestas del examen. Coadyuvó a recuperar la opinión del estudiante con discapacidad intelectual leve sobre el tipo de respuestas que se le presentan durante la aplicación del examen, los datos arrojaron la siguiente información:

Los estudiantes manifiestan que las respuestas de opción múltiple se encuentran estructuradas de manera compleja, lo que dificulta su comprensión, así también refieren que la similitud que existe entre estas, aunado a su extensión, los llega a confundir al momento de llevar a cabo la selección.

En respecto al formato de respuestas, los estudiantes consideran que las opciones de mayor dificultad son aquellas en las que tienen que relacionar números o letras, así como en las que se presentan la totalidad de texto en el segundo idioma: inglés.

R2) Técnica entrevista telefónica semiestructurada: Percepción del padre de familia sobre el proceso de evaluación CAED N. 065.

El instrumento guion de entrevista permitió conocer vía telefónica la opinión de seis padres de familia con hijos con algún tipo de discapacidad adscritos al CAED N. 065, los cuales se eligieron aleatoriamente, los resultados muestran:

En general los padres de familia entrevistados tienen una percepción positiva del servicio CAED N. 065, del que reportan a cubierto sus expectativas para que los 
estudiantes con algún tipo de discapacidad den continuidad en sus estudios en el nivel educativo medio superior, ya que mencionan prevalece un trato personalizado de los asesores adscritos al plantel hacia los estudiantes.

En respecto al proceso de evaluación para la acreditación modular reconocen se realiza por personal externo a la institución, dentro del cual se han percatado de algunas inconsistencias que afecta la acreditación de sus hijos, ya que estos han llegado a manifestar desmotivación, falta de interés o minusvalía hacia su persona para dar continuidad a sus estudios posterior a la no acreditación de algún examen en reiteradas ocasiones.

En este mismo sentido, los padres de familia refieren que sus hijos han realizado comentarios sobre extensión y complejidad del examen que presentan, dentro del cual prevalece una ausencia sobre la relación temática del libro de texto que se aborda durante asesoría con las preguntas que se plasman en el examen.

R3) Técnica Encuesta: Perspectiva del auxiliar y asesores del CAED N. 065 respecto al proceso de evaluación.

El instrumento tipo cuestionario se diseñó con base en un apartado I) Proceso de evaluación. La aplicación permitió recatar los comentarios del personal adscrito al CAED N. 065 equivalente a 3 personas encuestadas, las cuales corresponden a: 1: auxiliar administrativo y 2: asesores educativos. El análisis cualitativo de información respecto a este apartado muestra los siguientes resultados:

I) Proceso de evaluación. Permitió identificar que el personal conoce los lineamientos normativos y procedimentales de evaluación del CAED N. 065, dentro de los que reconoce que su función: se ciñe a la evaluación diagnóstica y formativa de apoyo al estudiante con discapacidad; y se ve limitada ante la intervención durante el proceso de evaluación por certificación que realiza el personal de Dirección General de Bachillerato (DGB).

En torno a la forma de evaluación hacia los estudiantes con discapacidad, el personal adscrito al CAED N. 065 argumenta que los exámenes que se aplican en dicho centro, son de carácter excluyente, debido a que no se encuentran adaptados a las 
características de los estudiantes con algún tipo de discapacidad, ya que refieren son exámenes escritos que se utilizan en el sistema de preparatoria abierta para evaluar a estudiantes sin discapacidad, lo que imposibilita a una persona con discapacidad visual responder a ellos de manera autónoma.

En lo que concierne a las dificultades que presenta el estudiante con discapacidad para la acreditación, el personal CAED N. 065 refiere el estudiante presenta rezago de conocimientos, asimismo se menciona que el contenido del examen difiere de los tópicos del libro que se revisan durante asesoría.

La opinión que el estudiante con algún tipo de discapacidad ha expresado a su asesor posterior a realizar un examen, afirman se encuentra relacionado a la dificultad de las preguntas para el desarrollo de problemas, la extensión de las lecturas o confusión en preguntas de relación que contienen números y letras, así como en uso de palabras que desconocen o la similitud existente en el tipo de respuestas que se presentan.

En relación a las consecuencias que produce la no acreditación del examen por certificación en el estudiante con algún tipo de discapacidad, se menciona por parte del personal que esta situación tiene repercusiones a nivel emocional y educativo, lo cual deriva en desmotivación y falta de interés por parte del este para continuar sus estudios, lo que causa baja temporal o definitiva del sistema educativo dentro del nivel media superior.

En respecto a la intervención del personal en el diseño, revisión, construcción de exámenes o reactivos, se menciona no participan, debido a que los exámenes son impuestos por la Secretaría de Educación Pública (SEP), no obstante, argumentan sería pertinente permitir a los asesores adaptar o simplificar los reactivos, integrar una escala para evaluar a los estudiantes o revisar el examen posterior a su presentación para trabajar sobre las áreas de oportunidad del estudiante.

\section{R3) Técnica encuesta: Evaluación Factorial de las Aptitudes Intelectuales Nivel 4 (EFAI-4)}

Los instrumentos tipo cuestionarios de la Evaluación Factorial de las Habilidades Intelectuales Nivel 4 (EFAI-4) permitió identificar las aptitudes de 20 estudiantes con discapacidad cognitiva leve a través de la aplicación de cinco pruebas: I) Aptitud espacial, 
II) Aptitud numérica, III) Razonamiento abstracto, IV) Aptitud verbal y V) Memoria. Los resultados respecto a cada subárea arrojaron puntuaciones por debajo de la media, bajo lo siguiente:

I) Aptitud espacial. Este apartado permite según Santamaría, Arribas, Pereña y Seisdedos (2005) conocer la capacidad del sujeto para llevar a cabo procesos mentales que impliquen imaginar, transformar o darles movimiento a los objetos, para llevar a cabo la solución de problemas. Los resultados de los estudiantes bajo esta condición denotaron una limitación para procesar información que les permita dar respuesta a problemas que impliquen trabajar con representaciones visuales como imágenes, gráficos o tablas que contengan información para analizar, comparar o transformar sus resultados en movimiento.

II) Aptitud numérica. De acuerdo con Santamaría et al (2005) este apartado muestra la capacidad del sujeto para razonar ante problemas de la vida diaria, que requieran el uso, manejo y análisis de datos numéricos. Los resultados muestran que la capacidad de los estudiantes bajo esta condición se encuentra limitada en aspectos que requieran llevar a cabo tareas que impliquen hacer uso de un razonamiento para interpretar de datos numéricos y dar solución a problemas cuyo contenido sea gráfico o requiera hacer una comparativa o relación numérica.

III) Razonamiento abstracto. Con base en Santamaría et al (2005) este apartado rescata la capacidad del sujeto para inducir o deducir a partir del razonamiento lógico, así como para resolver problemas complejos. Los resultados denotan que los estudiantes bajo esta condición muestran dificultad para realizar actividades que impliquen solucionar problemas con un alto grado de complejidad que requiera la secuenciación lógica de contenido, el uso de un razonamiento lógico para análisis, procesamiento y ejecución de tareas.

IV) Aptitud verbal. Con base en Santamaria et al (2005) este apartado coadyuva a visualizar la capacidad de comprensión y manejo de información verbal-escrita-oral del sujeto. Los resultados arrojaron que los estudiantes bajo esta condición muestran dificultad para la comprensión de argumentos y conceptos complejos que se le presentan de manera verbal, oral o escrita debido al escaso bagaje cultural de los 
mismos, así también reflejó una limitación para llevar a cabo expresiones de manera fluida ante terceras personas dentro de su contexto.

V) Memoria. Este apartado permite conocer de acuerdo con Santamaria et al (2005) la capacidad del sujeto para adquirir, retener y evocar información a mediano plazo. Los resultados arrojaron que los estudiantes bajo esta condición muestran una capacidad limitada para lograr apropiarse de algún tipo de información que se les presente de forma inmediata, ante la cual reflejan una limitante para poder recordar ciertos datos y hacer uso de estos en lapso determinado.

\section{Conclusiones}

La recuperación de datos cualitativos de las técnicas e instrumento aplicados permitió obtener información detallada para realizar la interpretación de la investigación desde la paralaje teórica y metodológica de paz integral sobre las condiciones de paz imposible inmersas en el proceso de evaluación por certificación dirigida a estudiantes con discapacidad intelectual leve adscritos al Centro de Atención a Estudiantes con Discapacidad (CAED) N. 065.

Los resultados de investigación se analizaron a partir del planteamiento de Sandoval (2018a) lo cual coadyuvó en el escenario práctico desde la parte empírica, a identificar la prevalencia y presencia de la violencia en su dimensión estructural, la cual subyuga en materia evaluativa, las garantías individuales y constitucionales del estudiante con discapacidad intelectual leve, a través de un marco de inequidad que se genera a partir de la aplicación de exámenes a gran escala para este sector poblacional en específico.

En este sentido, se generaron diversas conclusiones que de manera general coadyuvan a vislumbrar un panorama amplio sobre la situación de estudio bajo lo siguiente:

I) La perspectiva de los actores socioeducativos CAED N. 065: estudiantes con discapacidad intelectual leve; padres de familia; y personal adscrito a dicha institución, permite dilucidar que los lineamientos normativos en materia evaluativa como parte de las reformas estructurales de Estado, generan violencia estructural en tanto realicen evaluaciones mediante un estándar y criterio homogeneizante que limite el desarrollo académico del estudiante bajo esta condición. 
II) La presencia de violencia estructural a través de la evaluación externa se torna excluyente al momento de omitir la adecuación del instrumento tipo examen con base en las características propias del estudiante con discapacidad intelectual leve durante el proceso de evaluación, situación que se contrapone a la concreción de los CAED, los cuales se establecen bajo un marco de inclusión dirigidos a estudiantes con algún tipo de discapacidad y apoyo acorde a las necesidades de este, con la finalidad de garantizar su derecho constitucional a la educación.

III) El estudiante con discapacidad intelectual leve adscrito al CAED N. 065 requiere ser evaluado acorde al nivel cognitivo que presenta, con base en estándares prácticos y ejercicios sencillos que contemplen sus habilidades espaciales, numéricas, de razonamiento abstracto, verbales y de memoria.

IV) Se sugiere llevar a cabo una revisión teórica, metodológica y pedagógica para el diseño de reactivos acordes a la población con discapacidad intelectual leve adscritos al CAED N. 065, que responda a las necesidades y características estudiantiles durante el proceso de evaluación externa que se genera con fines de acreditación.

V) En síntesis, se establece que mientras prevalezca en CAED N. 065 un proceso de evaluación por certificación de carácter externo que evalúe el aprendizaje del estudiante con discapacidad intelectual leve bajo estándares homogéneos y en ausencia diversificada de criterios específicos dirigidos hacia esta población, la presencia de violencia estructural seguirá limitando el derecho a la educación del estudiante bajo esta condición, contribuyendo así, a la exclusión escolar derivada de la desigualdad de oportunidades en el sistema educativo media superior.

\section{Fuentes de consulta}

\section{Bibliográficas}

CASANOVA, A. (1998). La evaluación educativa. Escuela básica. España: Muralla

CASTILlO, A. Y CABRERIZO, D. (2010). Evaluación educativa de aprendizajes y competencias. España: Pearson.

DGB. (2017). Mecanismos Operativos del CAED. México: Autor

JARES, X. (1999). Educación para la paz. Su teoría y su práctica. (2nda) Ed. España: Popular 
LÓPEZ, S. E HINOJOSA., E. (2005). Evaluación del aprendizaje. Alternativas y Nuevos Desarrollos. México: Trillas.

SALAZAR, I. (2018a). Conflictos. pensares, interculturalidad para la paz y gestión en ambientes escolares. Venezuela: EAA.

SALAZAR, I. (2018b). No todas las violencias escolares son bullying, pero todas nos dañan. México: FOEM

SANDOVAL. E. (2018a). Etnografía e investigación acción intercultural para los conflictos y la paz. Metodologías Descolonizadoras. (2da) Ed. Venezuela: Alfonso Arena

SANDOVAL, E. (2016b). Educación para la paz integral. Memoria, interculturalidad y decolonialidad. Colombia: ARFO

SANTOS, M. (1995). La evaluación un proceso de diálogo, comprensión y mejora. ESPAÑA: ALJIBE

SANTAMARIA, P., ARRIBAS, D., PEREÑA, J., SEISDEDOS., N. (2005). Evaluación factorial de las habilidades intelectuales. Manual EFAI. Madrid: TEA

TOBÓN, S., PIMIENTA, J. Y GARCÍA, J. (2010) Secuencias didácticas: aprendizaje y evaluación de competencias. México: Prentice Hall

\section{Electrónicas}

CORTES, R., GONZÁLES, U., GUERRERO, J., Y RIVERA F. (2016). Informe estadístico sobre las evaluaciones en CAED. México: Autor. pp. 1-14. Recuperado de: https://drive.google.com/file/d/0B081hnOejChAR2hRX2RfTmNTZWs/view

DEL TORO, M. (2012). La Declaración Universal de Derechos Humanos: un texto multidimensional. México: CNDH. Recuperado de: http://appweb.cndh.org.mx/biblioteca/archivos/pdfs/fas_CSUPDH2-1aReimpr.pdf

DOF. (2017). Acuerdo número. 27/12/17 por el que se emiten las Reglas de Operación del Programa para la Inclusión y la Equidad Educativa para el ejercicio fiscal 2018. México: Secretaría de Servicios Parlamentarios pp. 1-94. Recuperado de: https://dof.gob.mx/nota_detalle.php?codigo=5509736\&fecha=29/12/2017

DOF. (2008). ACUERDO número 445 por el que se conceptualizan y definen para la Educación Media Superior las opciones educativas en las diferentes modalidades. México: Secretaría de Educación Pública pp. 1-7. Recuperado de: 
https://www.sep.gob.mx/work/models/sep1/Resource/7aa2c3ff-aab8-479f-ad93db49d0a1108a/a445.pdf

DOF. (2013). Programa Sectorial de Educación 2013-2018. México: Secretaría de Servicios Parlamentarios pp.1-57 Recuperado de: http://normatecainterna.sep.gob.mx/work/models/normateca/Resource/253/1/images /programa_sectorial_educacion_2013_2018.pdf

DOF. (2014). ACUERDO número 24/12/14 por el que se emiten las Reglas de Operación del Programa para la Inclusión y la Equidad Educativa para el ejercicio fiscal 2015. México: Secretaría de Educación Pública. Recuperado de: http://dof.gob.mx/nota_detalle.php?codigo=5377499\&fecha=27/12/2014

DOF. (2019) ACUERDO número 18/09/19 por el que se modifican las Reglas de Operación del Programa para la Inclusión y la Equidad Educativa para el ejercicio fiscal 2019, emitidas mediante diverso número 04/02/19, publicado el 28 de febrero de 2019. México: Secretaría de Educación Pública. Recuperado de: https://www.dof.gob.mx/nota_detalle.php?codigo=5573060\&fecha=20/09/2019

DOF. (2019). ACUERDO número 04/02/19 por el que se emiten las Reglas de Operación del Programa para la Inclusión y la Equidad Educativa para el ejercicio fiscal 2019. México: Secretaría de Educación Pública. Recuperado de: https://www.dof.gob.mx/nota_detalle.php?codigo=5551602\&fecha=28/02/2019

DOF. (2020) Programa Sectorial de Educación 2020-2024. México: Secretaría de Educación Pública. Recuperado de: https://www.dof.gob.mx/nota_detalle.php?codigo=5596202\&fecha=06/07/2020

INNE (2019) Cuadernillo técnico de evaluación educativa Nociones básicas en medición y evaluación en el contexto educativo. México: Autor. Recuperado de: https://www.inee.edu.mx/wp-content/uploads/2019/08/P2A251.pdf

MURILlO, J. (s/f) Teoría Fundamentada. Madrid: UAM Recuperado de: http://mastor.cl/blog/wp-content/uploads/2016/01/Teoriafundamentada.-Unasintesis.-pdf.pdf

PND. (2013-2018). México: Gobierno de la Republica pp.1-184. Recuperado de: https://www.snieg.mx/contenidos/espanol/normatividad/MarcoJuridico/PND_20132018.pdf 
PND. (2019). Programa Nacional de Desarrollo 2019-2024. México: Presidencia de la Republica. pp.1-64. Recuperado de: https://lopezobrador.org.mx/wpcontent/uploads/2019/05/PLAN-NACIONAL-DE-DESARROLLO-2019-2024.pdf

RAVELA, P. (2006). Fichas didácticas para comprender la evaluación educativa. Chile: CINDE.

Recuperado

de:

http://disde.minedu.gob.pe/bitstream/handle/123456789/4999/Fichas\%20did\%C3\% A1cticas\%20para\%20comprender\%201as\%20evaluaciones\%20educativas.pdf?sequ ence $=1 \&$ is Allowed $=\mathrm{y}$

SANDOVAL. E. (2013c). Etnografía para la paz, la interculturalidad y los conflictos. Revista de Ciencias Sociales (Cr), vol. III, núm. 141 pp. 11-24. Recuperado de: https://www.redalyc.org/pdf/153/15329875001.pdf 